\title{
Impact of an External Magnetic Field on the Shear Stresses Exerted by Blood Flowing in a Large Vessel
}

\author{
Agnès Drochon ${ }^{1 *}$, Manon Beuque ${ }^{2}$, Dima Abi-Abdallah Rodriguez ${ }^{3}$ \\ ${ }^{1}$ UMR CNRS 7338, Université de Technologie de Compiègne, Galileo Galilei Sorbonne Universités, Compiègne, France \\ ${ }^{2}$ Université de Technologie de Compiègne, Compiègne, France \\ ${ }^{3}$ IR4M, Université Paris Sud-Paris Saclay, Orsay, France \\ Email: *agnes.drochon@utc.fr
}

How to cite this paper: Drochon, A, Beuque, M. and Abi-Abdallah Rodriguez, D. (2017) Impact of an External Magnetic Field on the Shear Stresses Exerted by Blood Flowing in a Large Vessel. Journal of Applied Mathematics and Physics, 5, 14931502.

https://doi.org/10.4236/jamp.2017.57122

Received: June 10, 2017

Accepted: July 25, 2017

Published: July 28, 2017

Copyright (c) 2017 by authors and Scientific Research Publishing Inc. This work is licensed under the Creative Commons Attribution International License (CC BY 4.0).

http://creativecommons.org/licenses/by/4.0/

\begin{abstract}
The aim of this paper is to provide an advanced analysis of the shear stresses exerted on vessel walls by the flowing blood, when a limb or the whole body, or a vessel prosthesis, a scaffold... is placed in an external static magnetic field $B_{0}$. This type of situation could occur in several biomedical applications, such as magnetic resonance imaging (MRI), magnetic drug transport and targeting, tissue engineering, mechanotransduction studies... Since blood is a conducting fluid, its charged particles are deviated by the Hall effect, and the equations of motion include the Lorentz force. Consequently, the velocity profile is no longer axisymmetric, and the velocity gradients at the wall vary all around the vessel. To illustrate this idea, we expand the exact solution given by Gold (1962) for the stationary flow of blood in a rigid vessel with an insulating wall in the presence of an external static magnetic field: the analytical expressions for the velocity gradients are provided and evaluated near the wall. We demonstrate that the derivative of the longitudinal velocity with respect to the radial coordinate is preponderant when compared to the $\theta$-derivative, and that elevated values of $B_{0}$ would be required to induce some noteworthy influence on the shear stresses at the vessel wall.
\end{abstract}

\section{Keywords}

Magnetohydrodynamic Flow of Blood, Wall Shear Stresses, Magnetic Fields in Biomedical Applications

\section{Introduction}

The aim of this paper is to provide an advanced analysis of the shear stresses exerted on vessel walls by the flowing blood, when a limb or the whole body, or a 
vessel prosthesis, a scaffold... is placed in an external static magnetic field $B_{0}$.

As explained in [1] [2], such a situation may occur in several biomedical applications:

- magnetic resonance imaging (MRI) [3] [4] [5] [6] [7].

- magnetic drug transport and targeting [8]-[13]: magnetic particles containing or coated with therapeutics are injected into the bloodstream and concentrated to sites of disease under the influence of the magnetic field.

- tissue engineering [14] [15] [16] [17] [18]: magneto-responsive particles are guided by the magnetic force in order to enhance cellular invasion in the scaffolds.

- mechanotransduction studies and applications for regenerative medicine strategies (for example, with stem cells) [19] [20].

This analysis would also provide a risk assessment for the vessel wall (plaque rupture in case of atherosclerotic lesion [21], severity of some aneurysms [22], ...) or for other cells attachment and/or transmigration (white blood cells, tumor cells, cells seeded in vascular substitutes $[23], \ldots)$.

Since blood is a conducting fluid, its charged particles are deviated by the Hall effect thus inducing electrical currents and voltages along the vessel walls and in the neighboring tissues. The equations of motion include the Lorentz force $\mathbf{j}^{\wedge} \mathbf{B}$, where $\boldsymbol{j}$ is the electric current density. Consequently, the velocity profile is no longer axisymmetric, even in a cylindrical vessel; and the velocity gradients at the wall vary all around the vessel.

To illustrate this idea, we chose to expand the exact solution given by Gold [24] for the stationary flow of blood in a rigid vessel with an insulating wall in the presence of an external static magnetic field. This analysis completes previous ones [25] [26]. In the present paper, we provide the analytical expressions for the velocity gradients and evaluate them near the vessel wall.

\section{Unidirectional Steady Blood Flow in a Rigid Cylindrical Vessel with Insulating Walls}

As explained by Gold [24] and by Abi-Abdallah et al. [25], the Navier-Stokes equations including the Lorentz force (Equation (1)), coupled with the induction equation (Equation (2)) govern the flow of a conducting, incompressible, Newtonian fluid in an externally applied static magnetic field $\boldsymbol{B}_{0}$.

$$
\begin{gathered}
\rho\left(\frac{\partial \overrightarrow{\mathbf{u}}}{\partial t}+(\overrightarrow{\mathbf{u}} \cdot \vec{\nabla}) \overrightarrow{\mathbf{u}}\right)=-\operatorname{grad} P+\eta \Delta \overrightarrow{\mathbf{u}}+\frac{1}{\mu}(\operatorname{curl} \overrightarrow{\mathbf{B}}) \wedge \overrightarrow{\mathbf{B}} \\
\frac{\partial \overrightarrow{\mathbf{B}}}{\partial t}=\operatorname{curl}(\overrightarrow{\mathbf{u}} \wedge \overrightarrow{\mathbf{B}})+\frac{1}{\sigma \mu} \Delta \overrightarrow{\mathbf{B}}
\end{gathered}
$$

where $\mathbf{u}$ and $P$ are the fluid velocity and pressure; $\mu$ is the magnetic permeability; $\rho, \eta$ and $\sigma$ are the fluid density, viscosity and conductivity and the electric current density is expressed as $\mathbf{j}=(\operatorname{curl} \mathbf{B}) / \mu$.

Gold [24] then considered the case of a unidirectional steady blood flow in a rigid circular vessel with insulating walls and radius $R$ (Figure 1, [25] [26]). The 


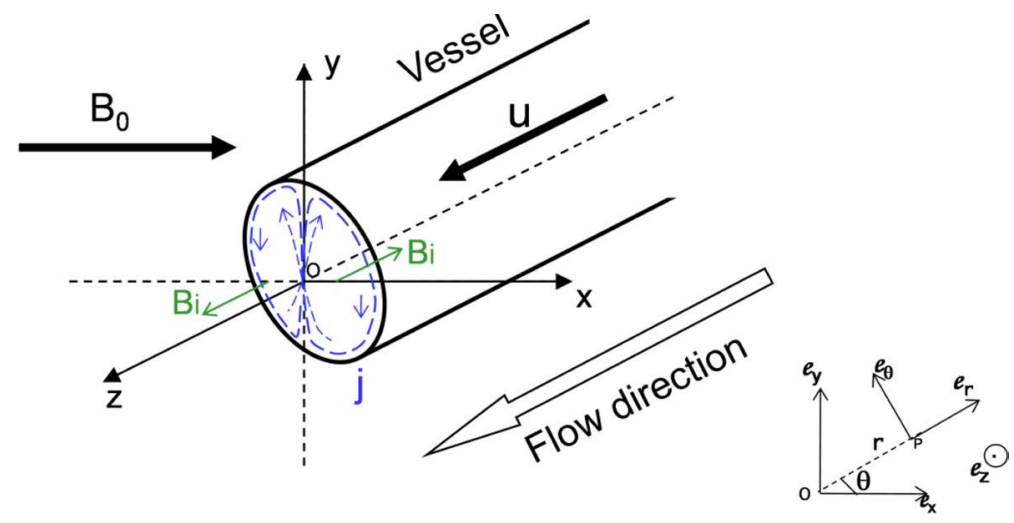

Figure 1. Schematic drawing of the studied problem (from [25]). The induced currents (blue dashed lines) are oriented along (Oy) in the tube center. Since they cannot escape the vessel (insulating walls), they return adjacent to the wall. Closed loops are thus generated, and these loops induce some magnetic field $B_{I}$ (Biot and Savart law). This induced field is parallel to the $\mathrm{Oz}$ axis with opposite directions on each side of $\mathrm{Oy}$.

velocity and magnetic field are defined in the cylindrical frame $\left(\mathbf{e}_{r}, \mathbf{e}_{\phi}, \mathbf{e}_{z}\right)$ as:

$$
\overrightarrow{\mathbf{u}} \mid \begin{gathered}
0 \\
0 \\
u_{z}(r, \theta)
\end{gathered} \text { and } \overrightarrow{\mathbf{B}} \mid \begin{aligned}
& B_{0} \cos \theta \\
& -B_{0} \sin \theta \\
& B_{I}(r, \theta)
\end{aligned}
$$

The induced magnetic field, $B_{D}$ is parallel to the flow and guarantees $\operatorname{div} \mathbf{B}=0$. The continuity equation $\operatorname{div} \mathbf{u}=0$ is also satisfied.

The longitudinal projections (along $\mathbf{e}_{z}$ ) of Equations ((1) and (2)) in the cylindrical frame are thus:

$$
\begin{gathered}
-\frac{\partial P}{\partial z}+\eta\left(\frac{1}{r} \frac{\partial u_{z}}{\partial r}+\frac{\partial^{2} u_{z}}{\partial r^{2}}+\frac{1}{r^{2}} \frac{\partial^{2} u_{z}}{\partial \theta^{2}}\right)+\frac{1}{\mu}\left(B_{0} \cos \theta \frac{\partial B_{I}}{\partial r}-\frac{B_{0} \sin \theta}{r} \frac{\partial B_{I}}{\partial \theta}\right)=0 \\
0=B_{0}\left(\cos \theta \frac{\partial u_{z}}{\partial r}-\frac{\sin \theta}{r} \frac{\partial u_{z}}{\partial \theta}\right)+\frac{1}{\sigma \mu}\left(\frac{1}{r} \frac{\partial B_{I}}{\partial r}+\frac{\partial^{2} B_{I}}{\partial r^{2}}+\frac{1}{r^{2}} \frac{\partial^{2} B_{I}}{\partial \theta^{2}}\right)
\end{gathered}
$$

The boundary conditions associated with this problem are:

$B_{I}(R, \theta)=0$ because the wall is non-conducting

and $u_{z}(R, \theta)=0$, because of the no-slip condition at the rigid wall

The equation system (4) to (6) is expressed in a non-dimensional form, using the following definitions:

$$
\tilde{U}=\frac{u_{z}}{u_{0}}, \quad \tilde{r}=\frac{r}{R}, \quad \tilde{B}=\frac{B_{I}}{B_{0}} \text {, and } G=-\frac{R^{2}}{\eta u_{0}} \frac{\partial P}{\partial z} \quad \text { (where } u_{0} \text { is some characteristic }
$$
mean velocity).

The numerical values of the different quantities are taken from Abi-Abdallah et al. [25]:

$$
\eta=4 \times 10^{-3} \mathrm{~Pa} \cdot \mathrm{s}, u_{0}=0.4 \mathrm{~m} / \mathrm{s}, \partial P / \partial \mathrm{z}=-128 \mathrm{~Pa} / \mathrm{m} \text {, and } R=0.01 \mathrm{~m} \text {; then }
$$

The non-dimensional solution given by Gold [24] for Equation (4) and (5), associated with the boundary conditions (6) is: 


$$
\tilde{B}(\tilde{r}, \theta)=\frac{\Re_{e m} G}{2 H_{a}^{2}}\left[E_{1}(\tilde{r}, \theta) A_{1}(\tilde{r}, \theta)-E_{2}(\tilde{r}, \theta) A_{2}(\tilde{r}, \theta)-2 \tilde{r} \cos \theta\right]
$$

and

$$
\left.\tilde{U}(\tilde{r}, \theta)=\frac{G}{2 H_{a}}\left[E_{1}(\tilde{r}, \theta)\right) A_{1}(\tilde{r}, \theta)+E_{2}(\tilde{r}, \theta) A_{2}(\tilde{r}, \theta)\right]
$$

with:

$$
\begin{gathered}
E_{1}(\tilde{r}, \theta)=\mathrm{e}^{-\frac{H_{a}}{2} \tilde{r} \cos \theta}, \\
A_{1}(\tilde{r}, \theta)=\alpha_{0} I_{0}\left(\frac{H_{a}}{2} \tilde{r}\right)+\sum_{n=1}^{\infty} 2 \alpha_{n} I_{n}\left(\frac{H_{a}}{2} \tilde{r}\right) \cos (n \theta), \\
E_{2}(\tilde{r}, \theta)=\mathrm{e}^{\frac{H_{a}}{2} \tilde{r} \cos \theta}, \\
A_{2}(\tilde{r}, \theta)=\alpha_{0} I_{0}\left(\frac{H_{a}}{2} \tilde{r}\right)+\sum_{n=1}^{\infty} 2(-1)^{n} \alpha_{n} I_{n}\left(\frac{H_{a}}{2} \tilde{r}\right) \cos (n \theta),
\end{gathered}
$$

and

$$
\alpha_{0}=\frac{I_{0}^{\prime}\left(\frac{H_{a}}{2}\right)}{I_{0}\left(\frac{H_{a}}{2}\right)} ; \quad \alpha_{n}=\frac{I_{n}^{\prime}\left(\frac{H_{a}}{2}\right)}{I_{n}\left(\frac{H_{a}}{2}\right)}
$$

The Hartmann number, $H_{a}$, is defined as: $H_{a}=B_{0} R \sqrt{\frac{\sigma}{\eta}}$, the magnetic Reynolds number, $R_{e m}$ as: $R_{e m}=R u_{0} \sigma \mu$, and the functions $I_{n}$ are the $n^{\text {th }}$ order modified Bessel functions of the first kind.

In order to evaluate the derivatives of the $I_{n}$ functions, the following identities are used:

$$
I_{0}^{\prime}(X)=I_{1}(X) \text { and } I_{n}^{\prime}(X)=I_{n-1}(X)-\frac{n}{X} I_{n}(X)
$$

In such flow configuration, the classical definition of the dimensional shear stresses would yield:

$$
\tau_{r z}=\eta \frac{\partial u_{z}}{\partial r} ; \quad \tau_{\theta z}=\frac{\eta}{r} \frac{\partial u_{z}}{\partial \theta}
$$

The corresponding non-dimensional expressions would be:

$\tilde{\tau}_{r z}=\frac{\partial \tilde{U}}{\partial \tilde{r}} ; \tilde{\tau}_{\theta z}=\frac{1}{\tilde{r}} \frac{\partial \tilde{U}}{\partial \theta}$, where $\tilde{\tau}_{r z}=\frac{\tau_{r z}}{\tau_{0}} ; \tilde{\tau}_{\theta z}=\frac{\tau_{\theta z}}{\tau_{0}}$, and $\tau_{0}=\frac{\eta u_{0}}{R} \quad\left(\tau_{0}=0.16 \mathrm{~Pa}\right.$, with the numerical data of this study).

It is thus necessary to calculate the velocity gradients (from Equation (8)). This can be done as follows:

$$
\begin{gathered}
\frac{\partial E_{1}}{\partial \tilde{r}}=-\frac{H_{a}}{2} \cos \theta \mathrm{e}^{-\frac{H_{a}}{2} \tilde{r} \cos \theta} \text { and } \frac{\partial E_{2}}{\partial \tilde{r}}=\frac{H_{a}}{2} \cos \theta \mathrm{e}^{\frac{H_{a} \tilde{r} \cos \theta}{2}} \\
\frac{\partial A_{1}}{\partial \tilde{r}}=\frac{H_{a}}{2}\left[\alpha_{0} I_{1}\left(\frac{H_{a}}{2} \tilde{r}\right)+\sum_{n=1}^{\infty} 2 \alpha_{n} \cos (n \theta)\left(I_{n-1}\left(\frac{H_{a}}{2} \tilde{r}\right)-\frac{2 n}{H_{a} \tilde{r}} I_{n}\left(\frac{H_{a}}{2} \tilde{r}\right)\right)\right]
\end{gathered}
$$




$$
\begin{gathered}
\frac{\partial A_{2}}{\partial \tilde{r}} \\
=\frac{H_{a}}{2}\left[\alpha_{0} I_{1}\left(\frac{H_{a}}{2} \tilde{r}\right)+\sum_{n=1}^{\infty} 2(-1)^{n} \alpha_{n} \cos (n \theta)\left(I_{n-1}\left(\frac{H_{a}}{2} \tilde{r}\right)-\frac{2 n}{H_{a} \tilde{r}} I_{n}\left(\frac{H_{a}}{2} \tilde{r}\right)\right)\right] \\
\frac{\partial E_{1}}{\partial \theta}=\frac{H_{a}}{2} \tilde{r} \sin \theta \mathrm{e}^{-\frac{H_{a}}{2} \tilde{r} \cos \theta} \text { and } \frac{\partial E_{2}}{\partial \theta}=-\frac{H_{a}}{2} \tilde{r} \sin \theta \mathrm{e}^{\frac{H_{a}}{2} \tilde{r} \cos \theta} \\
\frac{\partial A_{1}}{\partial \theta}=-\sum_{n=1}^{\infty} 2 n \alpha_{n} I_{n}\left(\frac{H_{a}}{2} \tilde{r}\right) \sin (n \theta) \text { and } \frac{\partial A_{2}}{\partial \theta}=\sum_{n=1}^{\infty} 2 n(-1)^{n+1} \alpha_{n} I_{n}\left(\frac{H_{a}}{2} \tilde{r}\right) \sin (n \theta)
\end{gathered}
$$

Gathering all, one obtains:

$$
\frac{\partial \tilde{U}}{\partial \tilde{r}}=\frac{G}{2 H_{a}}\left[\frac{\partial E_{1}}{\partial \tilde{r}} A_{1}+E_{1} \frac{\partial A_{1}}{\partial \tilde{r}}+\frac{\partial E_{2}}{\partial \tilde{r}} A_{2}+E_{2} \frac{\partial A_{2}}{\partial \tilde{r}}\right]
$$

and:

$$
\frac{\partial \tilde{U}}{\partial \theta}=\frac{G}{2 H_{a}}\left[\frac{\partial E_{1}}{\partial \theta} A_{1}+E_{1} \frac{\partial A_{1}}{\partial \theta}+\frac{\partial E_{2}}{\partial \theta} A_{2}+E_{2} \frac{\partial A_{2}}{\partial \theta}\right]
$$

\section{Results}

The term $\frac{\partial \tilde{U}}{\partial \tilde{r}}(1, \theta), 0<\theta<2 \pi$, represents the change of velocity in the radial direction, all around the vessel wall.

The term $\frac{\partial \tilde{U}}{\partial \theta}(\tilde{r}, \theta)$ represents the change of velocity in the azimuthal direction, at a given value of $\tilde{r}$. The velocity is zero everywhere at the wall $(r=R)$; consequently the velocity gradient $\frac{\partial \tilde{U}}{\partial \theta}(1, \theta)$ is also zero. The interesting quantities are thus $\frac{\partial \tilde{U}}{\partial \theta}(\tilde{r}, \theta)$, for $\tilde{r}$ close to 1 , but lower than 1 .

The dependence of the non-dimensional velocity $\tilde{U}$ upon $\theta$ (for $-\pi<\theta<+\pi$ ) is presented in Figure 2. It has been computed for $\tilde{r}=0.5$. In the absence of magnetic field $\left(H_{a}=0\right)$, the situation is axisymmetric and the velocity does not depend upon $\theta$. The flow is the classical Poiseuille flow and, as expected, $\tilde{U}(\tilde{r}=0.5)=3 / 2$. When the Hartmann number increases, the flow is furthermore reduced (this is the decelerating effect due to the Lorentz force), and the dependence upon $\theta$ (asymmetry of the flow) is more and more pronounced. The velocity is maximal in the direction $\theta=0$ and $\theta=\pi$ (or $-\pi$ ), according to the fact that the profile is flattened and stretched parallel to the direction of $B_{0}$ (along Ox) [25]. For the same reason, the velocity is minimal in the direction $\theta=\pi / 2$ or $-\pi / 2$.

The same type of results is shown in Figure 3, where the dependence of the velocity upon $\theta$ has been illustrated at $\tilde{r}=0.99$ (near the vessel wall). Of course, the velocities are very small, since at the wall, they are exactly zero. As in Figure 2 , we can observe that the curve obtained for the case $H_{a}=0.16$ is superimposed with the curve $H_{a}=0$, meaning that the influence of a magnetic field $B_{0}=1.5 \mathrm{~T}$ (corresponding to $H_{a}=0.16$ ) remains negligible. Moreover, when $H_{a}=0$, the 


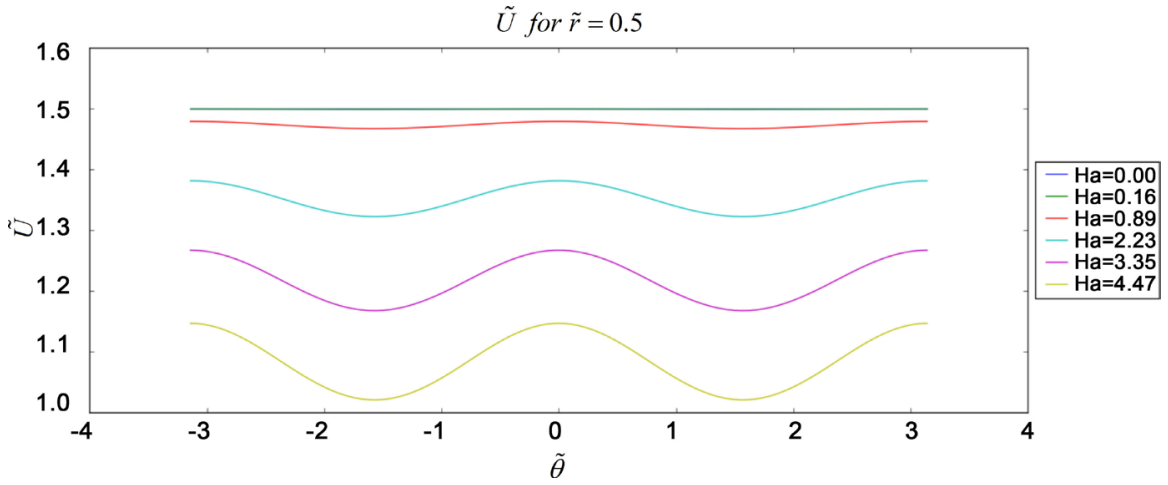

Figure 2. Dependence of the non-dimensional velocity $\tilde{U}$ upon $\theta$ (for $-\pi<\theta<+\pi$ ), at $\tilde{r}=0.5$.

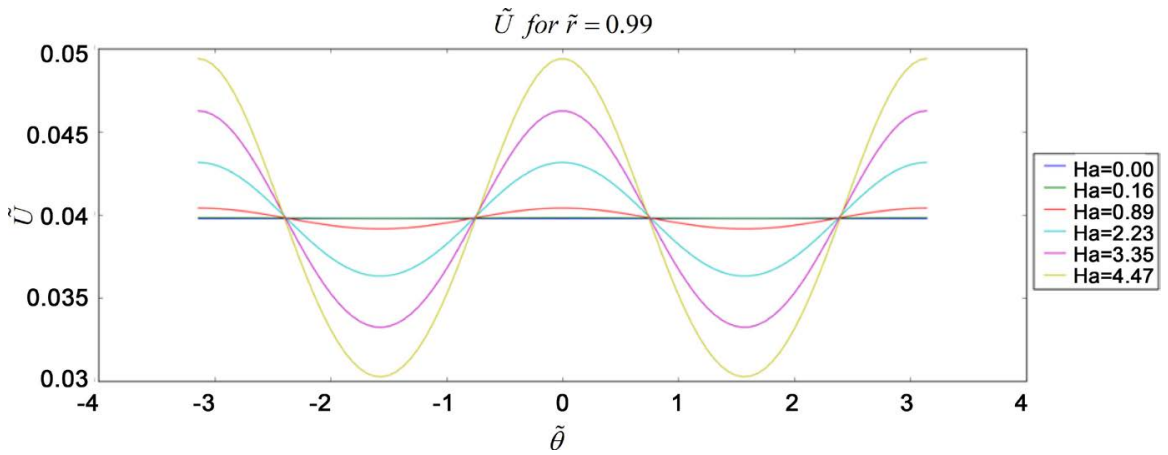

Figure 3. Dependence of the non-dimensional velocity $\tilde{U}$ upon $\theta$ (for $-\pi<\theta<+\pi$ ), at $\tilde{r}=0.99$

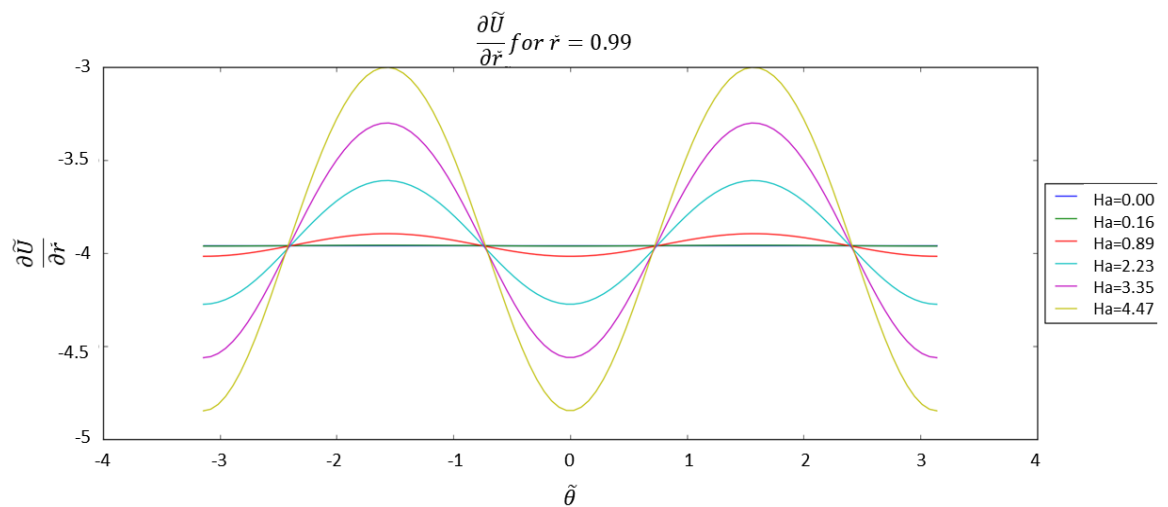

Figure 4. Dependence of the non-dimensional velocity gradient $\partial \tilde{U} / \partial \tilde{r}$ upon $\theta$ (for $-\pi<\theta<+\pi)$, at $\tilde{r}=0.99$.

value obtained for the non-dimensional velocity at $r=0.99{ }^{\star} R$ is 0.0398 , which is the Poiseuille value.

The dependence of the non-dimensional velocity gradient $\partial \tilde{U} / \partial \tilde{r}$ upon $\theta$ (for $-\pi<\theta<+\pi$ ), at $\tilde{r}=0.99$ is illustrated in Figure 4 and at $\tilde{r}=1$ in Figure 5 . These gradients are negative, since the value of the velocity $\tilde{U}$ decreases when going towards the vessel wall $(\tilde{U}=0$, when $\tilde{r}=1)$. As previously noted, the influence of a $1.5 \mathrm{~T}$ magnetic field $\left(H_{a}=0.16\right)$ is not discernible, and the absolute values of the gradients $\partial \tilde{U} / \partial \tilde{r}$ are maximum for $\theta=0$, and $\theta=\pi$, or $-\pi$ 
(due to the fact that the profile is stretched along Ox).

In the absence of a magnetic field $\left(H_{a}=0\right)$, the Poiseuille value $(\partial \tilde{U} / \partial \tilde{r}=-4$, at the wall) is obtained, and no dependence upon $\theta$ is observed (axisymmetric situation). The maximum values of $\partial \tilde{U} / \partial \tilde{r}$ are increased by about $25 \%$ in the case of a very strong magnetic field $\left(H_{a}=4.47, B_{0}=40 \mathrm{~T}\right)$, when compared to the case $H_{a}=0$.

The dependence of the non-dimensional velocity gradient $\partial \tilde{U} / \partial \theta$ upon $\theta$ (for $-\pi<\theta<+\pi$ ), at $\tilde{r}=0.5$ and at $\tilde{r}=0.99$ is illustrated in Figure 6 and Figure 7 respectively. Since we have $\tilde{U}=0$, everywhere at the vessel wall $(\tilde{r}=1)$, we also have $\partial \tilde{U} / \partial \theta=0$, for $\tilde{r}=1$. Consequently, the absolute values of the $\partial \tilde{U} / \partial \theta$ velocity gradients decrease when $\tilde{r}$ tends towards 1 .

In the absence of a magnetic field $\left(H_{a}=0\right)$, the situation is axisymmetric, and there is no dependence upon $\theta$.

The non-dimensional shear stress, $\tilde{\tau}_{\theta z}$, could be obtained dividing $\partial \tilde{U} / \partial \theta$ by the value of the corresponding $\tilde{r}$ (Equation (11)). For example, if we look at the maximum value of $\tilde{\tau}_{\theta z}$ for $\tilde{r}=0.99$, we obtain $\left|\tilde{\tau}_{\theta z}\right|<0.02$, which is negligible when compared to $\tilde{\tau}_{r z}$ (Figure 4).

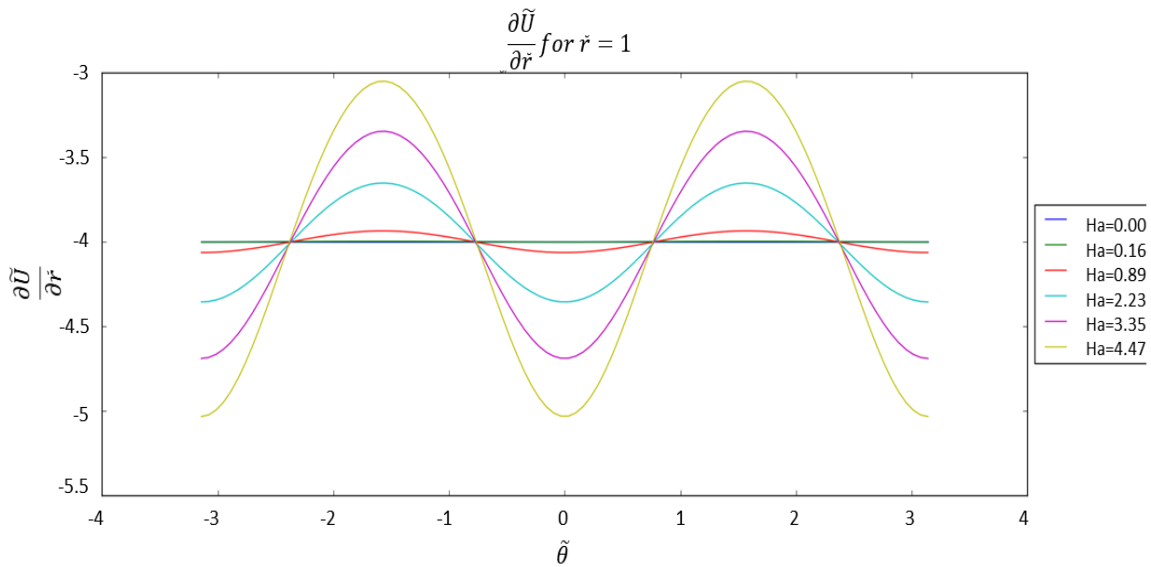

Figure 5. Dependence of the non-dimensional velocity gradient $\partial \tilde{U} / \partial \tilde{r}$ upon $\theta$ (for $-\pi<\theta<+\pi)$, at $\tilde{r}=1$.

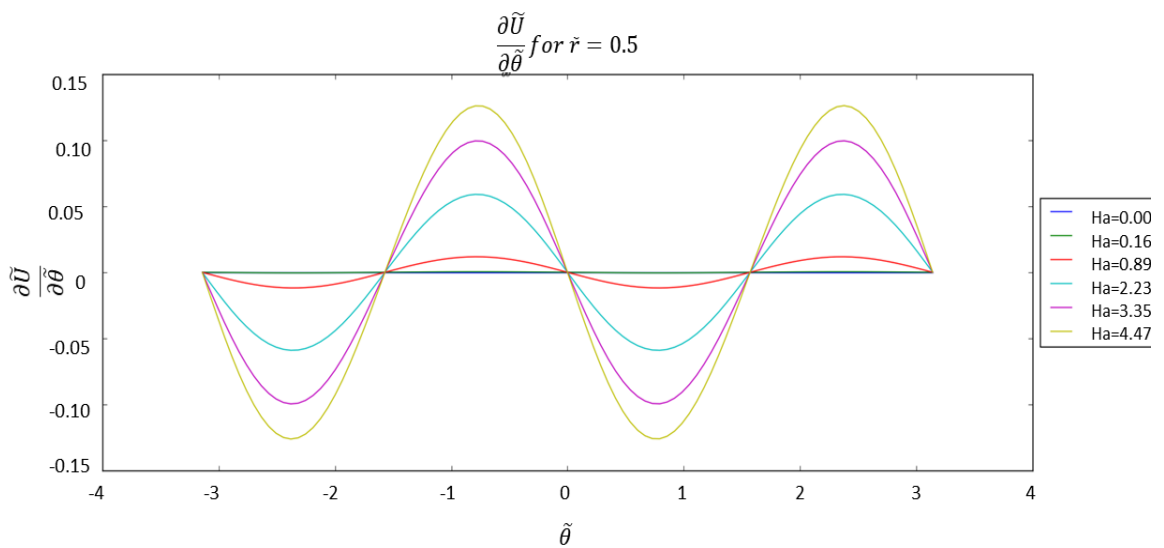

Figure 6. Dependence of the non-dimensional velocity gradient $\partial \tilde{U} / \partial \theta$ upon $\theta$ (for $-\pi<\theta<+\pi)$, at $\tilde{r}=0.5$. 


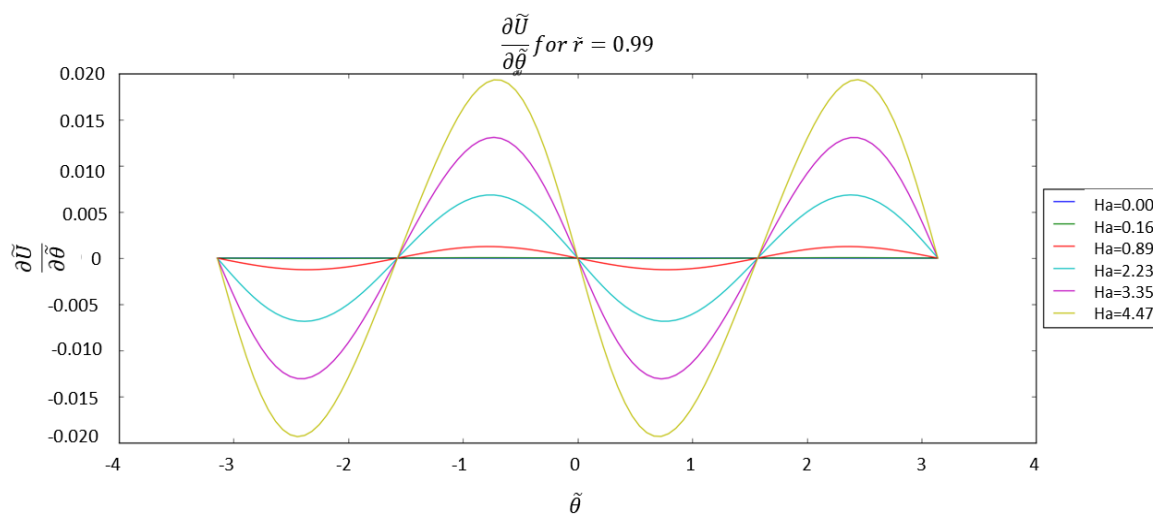

Figure 7. Dependence of the non-dimensional velocity gradient $\partial \tilde{U} / \partial \theta$ upon $\theta$ (for $-\pi<\theta<+\pi)$, at $\tilde{r}=0.99$.

\section{Conclusion}

In this paper, we demonstrate that the quantities $\tilde{\tau}_{r z}=\frac{\partial \tilde{U}}{\partial \tilde{r}}$ and $\tilde{\tau}_{\theta z}=\frac{1}{\tilde{r}} \frac{\partial \tilde{U}}{\partial \theta}$ both depend upon $\theta$, but that this dependence may be considered negligible for low values of $B_{0}\left(B_{0}<3 \mathrm{~T}\right)$. We also demonstrate that, at the vessel wall, $\tilde{\tau}_{\theta z}$ is several orders of magnitude smaller than $\tilde{\tau}_{r z}$, and that, in the presence of a very strong magnetic field $\left(H_{a}=4.47, B_{0}=40 \mathrm{~T}\right)$, the maximum value of $\tilde{\tau}_{r z}$ is only increased by $25 \%$, when compared to its value in the absence of a magnetic field $\left(H_{a}=0\right)$. Consequently, in most of the situations encountered in biomedical applications, the classical calculation $(\eta(\partial u / \partial r))$ remains a good approximation to evaluate the shear stresses at the wall.

\section{Declarations}

Competing interests: none.

Funding: none.

Ethical approval: not required.

\section{References}

[1] Gregory, T.S., Cheng, R., Tang, G., Mao, L. and Tse, Z. (2016) The Magnetohydrodynamic Effect and Its Associated Material Designs for Biomedical Applications: A State-of-the-Art Review. Advanced Functional Materials, 26, 3942-3952. https://doi.org/10.1002/adfm.201504198

[2] Drochon, A. (2016) Sinusoïdal Flow of Blood in a Cylindrical Deformable Vessel Exposed to an External Magnetic Field. The European Physical Journal Applied Physics, 73, 31101. https://doi.org/10.1051/epjap/2016150530

[3] Laffon, E., Marthan, R., Montaudon, M., Latrabe, V., Laurent, F. and Ducassou, D. (2005) Feasibility of Aortic Pulse Pressure and Pressure Wave Velocity MRI Measurement in Young Adults. Journal of Magnetic Resonance Imaging, 21, 53-58. https://doi.org/10.1002/jmri.20227

[4] Ibrahim, E.S., Johnson, K., Miller, A., Shaffer, J. and White R. (2010) Measuring Aortic Pulse Wave Velocity using High-Field Cardiovascular Magnetic Resonance: Comparison of Techniques. Journal of Cardiovascular Magnetic Resonance, 12, 2639. https://doi.org/10.1186/1532-429X-12-26 
[5] Nijm, G., Swiryn, S., Larson, A. and Sahakian A. (2008) Extraction of the Magnetohydrodynamic Blood Flow Potential from the Surface Electrocardiogram in Magnetic Resonance Imaging. Medical \& Biological Engineering \& Computing, 46, 729733. https://doi.org/10.1007/s11517-008-0307-1

[6] Kyriakou, A., Neufeld, E., Szczerba, D., Kainz, W., Luechinger, R., Kozerke, S., Mc Gregor, R. and Kuster, N. (2012) Patient-Specific Simulations and Measurements of the Magneto-Hemodynamic Effect in Human Primary Vessels. Physiological Measurement, 33, 117-130. https://doi.org/10.1088/0967-3334/33/2/117

[7] Krug, K., Rose, G., Clifford, G. and Oster, J. (2013) ECG-Based Gating in Ultra High Field Cardiovascular Magnetic Resonance Using an Independent Component Analysis Approach. Journal of Cardiovascular Magnetic Resonance, 15, 104-116. https://doi.org/10.1186/1532-429X-15-104

[8] Nacev, A., Beni, C., Bruno, O. and Shapiro B. (2010) Magnetic Nanoparticle Transport within Flowing Blood and into Surrounding Tissue. Nanomedicine, 5, 1459 1466. https://doi.org/10.2217/nnm.10.104

[9] Kenjeres, S. (2008) Numerical Analysis of Blood Flow in Realistic Arteries Subjected to Strong Non-Uniform Magnetic Fields. International Journal of Heat and Fluid Flow, 29, 752-764.

[10] Ciofani, G., Riggio, C., Raffa, V., Menciassi, A. and Cuschieri A. (2009) A Bi-Modal Approach against Cancer: Magnetic Alginate Nanoparticles for Combined Chemotherapy and Hyperthermia. Medical Hypotheses, 73, 80-82. https://doi.org/10.1016/j.mehy.2009.01.031

[11] Morega, A., Dobre, A. and Morega, M. (2011) Magnetic Field-Flow Interactions in Drug Delivery through an Arterial System. Revue Roumaine des Sciences Techniques-Serie Electrotechnique et Energetique, 56, 199-208.

[12] Riegler, J., Lau, K., Garcia-Prieto, A., Price, A., Richards, T., Pankhurst, Q. and Lythgoe, M. (2011) Magnetic Cell Delivery for Peripheral Arterial Disease: A Theoretical Framework. Medical Physics, 38, 3932-3943. https://doi.org/10.1118/1.3593363

[13] Sensenig, R., Sapir, Y., MacDonald, C., Cohen, S. and Polyak, B. (2012) Magnetic Nanoparticle-Based Approaches to Locally Target Therapy and Enhance Tissue Regeneration in Vivo. Nanomedecine, 7, 1425-1442.

https://doi.org/10.2217/nnm.12.109

[14] Perea, H., Aigner, J., Hopfner, U. and Wintermantel, E. (2006) Direct Magnetic Tubular Cell Seeding: A Novel Approach for Vascular Tissue Engineering. Cells Tissues Organs, 183, 156-165. https://doi.org/10.1159/000095989

[15] Perea, H., Aigner, J., Heverhagen, J.T., Hopfner, U. and Wintermantel E. (2007) Vascular Tissue Engineering with Magnetic Nanoparticles: Seeing Deeper. Journal of Tissue Engineering and Regenerative Medicine, 1, 318-321. https://doi.org/10.1002/term.32

[16] Xu, H., Othman, S. and Magin, R. (2008) Monitoring Tissue Engineering Using Magnetic Resonance Imaging. Journal of Bioscience and Bioengineering, 106, 515-527. https://doi.org/10.1263/jbb.106.515

[17] Poirier-Quinot, M., Frasca, G., Wilhelm, C., Luciani, N., Ginefri, J.C., Darasse, L., Letourneur, D., Visage, C. and Gazeau, F. (2010) High Resolution 1.5 Tesla Magnetic Resonance Imaging for Tissue-Engineered Constructs: A Noninvasive Tool to Assess Three-Dimensional Scaffold Architecture and Cell Seeding Tissue Engineering Part C: Methods, 16, 185-200.

[18] Sasaki, T., Iwasaki, N., Kohno, K., Kishimoto, M., Majima, T., Nishimura, S.I. and Minami, A. (2008) Magnetic Nanoparticles for Improving Cell Invasion in Tissue 
Engineering. Journal of Biomedical Materials Research, 86A, 969-978. https://doi.org/10.1002/jbm.a.31724

[19] Cartmell, S.H. and Dobson, J. (2011) The Use of Magnetic Particles in Tissue Engineering. In: Biju, V., Mundayoor, S., Anas, A. and Ishikawa, M., Eds., Nanotechnologies for the Life Sciences, Wiley, New York.

[20] Santos, L., Reis, R. and Gomes, M. (2015) Harnessing Magnetic-Mechano Actuation in Regenerative Medicine and Tissue Engineering. Trends in Biotechnology, 33, 471-479. https://doi.org/10.1016/j.tibtech.2015.06.006

[21] Weddell, J., Kwack, J., Imoukhuede, P. and Masud, A. (2015) Hemodynamic Analysis in an Idealized Artery Tree: Differences in Wall Shear Stress between Newtonian and Non-Newtonian Blood Models. PLoS One, 10, e0124575.

https://doi.org/10.1371/journal.pone.0124575

[22] Boussel, L., Rayz, V., Martin, A., Acevedo-Bolton, G., Lawton, M., Higashida, R., Smith, W., Young, W. and Saloner, D. (2009) Phase-Contrast MRI Measurements in Intra-Cranial Aneurysms In-Vivo of Flow Patterns, Velocity Fields and Wall Shear-Stress: A Comparison with CFD. Magnetic Resonance in Medicine, 61, 409417. https://doi.org/10.1002/mrm.21861

[23] Castro, E. and Mano, J. (2013) Magnetic Force-Based Tissue Engineering and Regenerative Medicine. Journal of Biomedical Nanotechnology, 9, 1129-1136. https://doi.org/10.1166/jbn.2013.1635

[24] Gold, R. (1962) Magneto Hydrodynamic Pipe Flow. Journal of Fluid Mechanics, 13, 505-512. https://doi.org/10.1017/S0022112062000889

[25] Abi-Abdallah, D., Drochon, A., Robin, V. and Fokapu, O. (2009) Effects of Static Magnetic Field Exposure on Blood Flow. European Physical Journal Applied Physics, 45, Article ID: 11301. https://doi.org/10.1051/epjap:2008193

[26] Drochon, A., Robin, V., Fokapu, O. and Abi-Abdallah, R.D. (2016) Stationary Flow of Blood in a Rigid Vessel in the Presence of an External Magnetic Field: Considerations about the Forces and Wall Shear Stresses. Applied Mathematics, 7, 130-136. https://doi.org/10.4236/am.2016.72012

Scientific Research Publishing

Submit or recommend next manuscript to SCIRP and we will provide best service for you:

Accepting pre-submission inquiries through Email, Facebook, LinkedIn, Twitter, etc. A wide selection of journals (inclusive of 9 subjects, more than 200 journals)

Providing 24-hour high-quality service

User-friendly online submission system

Fair and swift peer-review system

Efficient typesetting and proofreading procedure

Display of the result of downloads and visits, as well as the number of cited articles

Maximum dissemination of your research work

Submit your manuscript at: http://papersubmission.scirp.org/

Or contact jamp@scirp.org 